\title{
El cultivo de alfalfa utilizando agua de perforación, agua residual urbana y precipitaciones
}

\author{
José 0. Plevich ${ }^{1}$, Angel R. S. Delgado $^{2}$, Cecilia Saroff $^{1}$, Juan C. Tarico ${ }^{1}$, \\ R. J. Crespi ${ }^{3} \& 0$ mar M. Barotto ${ }^{1}$
}

\begin{abstract}
RESU MEN
El objetivo de este trabajo fue analizar la producción de biomasa aérea, la eficiencia del uso de agua y el valor nutritivo de alfalfa cultivada aplicando riego con agua residual urbana, agua de perforación y un testigo (precipitaciones), en el Campus de la Universidad $\mathrm{N}$ acional de Rio Cuarto (Córdoba-Argentina). Los tratamientos de riego tuvieron un efecto positivo sobre la producción de biomasa aérea obteniéndose un $24 \%$ más de producción de biomasa que en la situación de secano (precipitaciones). Entre los tratamientos con riego también se manifestaron diferencias estadísticamente significativas. La alfalfa regada con aguas residuales urbana supero en un $19 \%$ a la cultivada con agua de perforación. La eficiencia del uso del agua por parte del cultivo se incrementó cuando el riego se realizó con aguas residuales urbanas. Además, se determinó que la alfalfa regada con agua residual, supera los valores nutritivos del cultivo que crece en condiciones sin riego; encontrándose 39\% más de proteínas, $14 \%$ más de digestibilidad e igual porcentaje de energía metabólica. Se puede concluir que el agua residual urbana representa otra posible fuente de agua, factible de ingresar al suelo y estar a disposición para el cultivo de alfalfa.
\end{abstract}

Palabras-clave: Medicago sativa L, tratamiento de agua, filtros verdes, biomasa, calidad ambiental

\section{The cultivation of alfalfa using artesian well water, urban wastewater and rainfall}

\begin{abstract}
A B STRACT
The objective of this study was to analyse the production of alfalfa biomass, the efficiency of water use and the nutritional value of forage, inside an irrigation structure of an experimental facility that uses treated urban wastewater, artesian well water and rainfall, at the campus of the U niversidad National de Rio Cuarto (Córdoba-Argentina). The irrigation treatment had a positive effect on the production of biomass of alfalfa. On an average $24 \%$ increase in production was observed when compared to dry-land farming. Regarding the quality of the irrigation water, statistical differences in production were observed, the irrigation with urban wastewater produced $19 \%$ more than that of well water. The efficiency of the water use by the crop increased when the irrigation was done using urban wastewater. The alfalfa irrigated with wastewater surpasses the nutritional values of the crop in normal field conditions: $39 \%$ more proteins, $14 \%$ more digestibility and equal percentage of metabolic energy. It is concluded that the urban wastewater represents possible water source for the alfalfa crop.
\end{abstract}

Key words: Medicago sativa L., water treatment, green filter, biomass, environment quality

\footnotetext{
${ }^{1}$ Depto de Producción Vegetal/U niversidad Nacional de Río Cuarto, Ruta Nac 36, Km 601, Río Cuarto, Córdoba, Argentina. Te: 054358 4676509. E-mail: oplevich@ayv.unrc.edu.ar; csaroff@ayv.unrc.edu.ar; juanct82@hotmail.com; obarotto@ayv.unrc.edu.ar

2 U FRRJ/PPGCTIA, BR 465, Km. 7, Seropédica, CEP 2385100, Rio de Janeiro, Brasil. E-mail: asanchez@ufrrj.br

${ }^{3}$ Depto de Ecologia Agrícola/U niversidad N acional de Río Cuarto. Te: 054358 4676199. E-mail: rcrespi@ayv.unrc.edu.ar
} 


\section{INTRODUCCIÓN}

Sin duda que la contaminación del agua es uno de los problemas ambientales más importante a nivel mundial. A ello se agrega el daño irreparable que las aguas residuales no tratadas infligen en el ambiente natural, ríos, lagos, acuíferos degradados irreversiblemente, ecosistemas, especies animales y vegetales desaparecidas; entre otras.

Estos perjuicios repercuten inevitablemente en las poblaciones que habitan en las zonas degradadas, generándose un impacto creciente en la salud y calidad de vida en general.

El tratamiento y reciclaje de aguas servidas, es un factor importante al considerar que de esta forma se reduce la presión sobre la disponibilidad y calidad del agua, además de minimizar la descarga de aguas servidas sin tratamiento a los ríos (Leite et al., 2005; Pereira et al., 2009; Santos et al., 2010; Maggi et al., 2011). Sistemas que usan aguas residuales son posibles de implementar y utilizar, siempre y cuando consideren técnicas que permiten evitar la transmisión de infecciones relacionadas con el origen de las mismas.

El empleo de aguas residuales es posible a través del tratamiento que usa tecnologías no convencionales como los filtros verdes y lagunas de estabilización. Los filtros verdes con probada eficiencia en la utilización de estas aguas, pueden remover altas concentraciones de residuos a través de un mecanismo de fitorremediación (Schnoor et al., 1995; Santos et al., 2000).

Las lagunas de estabilización constan de una pileta (o una serie de ellas), en las cuales se depositan los efluentes para ser tratados biológicamente. Generalmente los efluentes pasan primero por una laguna anaeróbica (de mayor profundidad) y luego por una laguna aeróbica (de menor profundidad); esta última conocida como de maduración. El agua permanece entre cuatro y siete horas en el denominado tanque de aeración y durante ese tiempo, las bacterias degradan la materia orgánica. En la degradación se producen más microorganismos, $\mathrm{CO}_{2}$, $\mathrm{H}_{2} \mathrm{O}$ y cantidades variables de otros productos (Souza et al., 2010). Fundamentalmente se coloca en estrecho contacto, las aguas a ser tratadas y el lodo cargado de una elevada concentración de microorganismos, que con un aire burbujeando continuamente, permite alcanzar suficiente cantidad de oxígeno en este proceso de reutilización del agua.

En la Universidad Nacional de Río Cuarto (UNRC), CórdobaArgentina; para evitar la contaminación de las napas freáticas con el agua residual generada por las residencias estudiantiles, se encuentra instalada una planta experimental de tratamiento de agua residual, que usa filtros verdes y lagunas de estabilización. En la experiencia aquí descrita, se trabajó con un filtro verde de alfalfa (Medicago sativa L.). Existen trabajos recientes que tratan con crecimiento y producción de culturas utilizando aguas residuales (Silva et al., 2011).

La reutilización de efluentes tratados se ha incrementado en la agricultura, ya que tiene como metas la preservación de las escasas fuentes de agua en la región, así como la manutención de la calidad ambiental. Todo esto procurando una agricultura sustentable. Básicamente consiste en la aplicación del agua residual tratada en la producción de determinadas culturas (ajo, cebolla o alfalfa, por ejemplo), y en cualquier caso, se hace necesario conocer la fisiología del cultivo, la tierra disponible y la matriz del suelo para su tratamiento (Álvarez et al., 2002; Cabral et al., 2011).

La alfalfa es la principal especie forrajera de la Argentina y base en la producción de carne y leche de la región pampeana (Basigalup et al., 2007). De acuerdo a Collino et al. (2005), la provincia de Córdoba requiere entre 1.100 y $1.550 \mathrm{~mm}$ de agua para obtener una producción potencial de alfalfa para forraje. Considerando que en Río Cuarto, y en general en el sur de Córdoba, las precipitaciones son deficitarias, hallamos interesante la realización de una experiencia que combinase el uso de un filtro verde con la producción de biomasa forrajera y de esta manera estaríamos contribuyendo con la depuración del agua residual urbana de las residencias estudiantiles de la UNRC y con la producción de reservas de rollos o fardos de alfalfa que pueden ser destinados al período invernal donde existe escasez de forraje.

El propósito de este trabajo fue analizar la producción de alfalfa (biomasa aérea), la eficiencia del uso de agua durante el ciclo de cultivo y el valor nutritivo del forraje obtenido; dentro de una estructura de riego que usa agua residual urbana tratada, agua de perforación y solo agua de precipitaciones.

\section{MATERIAL Y METODOS}

El experimento fue desarrollado en el Campus de la Universidad Nacional de Río Cuarto (Córdoba-Argentina), próximo a las Residencias Estudiantiles Universitarias (REU), ubicado a $33^{\circ} 07^{\prime}$ LS, 64 $4^{\circ} 14^{\prime}$ LO y a $421 \mathrm{~m}$ sobre el nivel del mar; donde existe una planta experimental para el tratamiento y reutilización de aguas residuales urbanas. El experimento se monto sobre un filtro verde experimental constituido por alfalfa (Medicago sativa L. variedad Imperial 6) de dos años de edad. Sobre una superficie de $810 \mathrm{~m}^{2}$ se establecieron bloques y sobre estos se distribuyeron aleatoriamente los tratamientos en parcelas: 1) Riego con agua residual urbana 2) Riego con agua de perforación y 3) secano (agua solo precipitaciones). De esta manera quedo constituido un diseño de bloques completamente al azar, con 9 bloques y 27 parcelas. Sobre estas parcelas de $30 \mathrm{~m}^{2}$ de superficie, se evaluó la producción de biomasa, la evapotranspiración, la eficiencia en el uso del agua y el valor nutritivo del cultivo de alfalfa.

Para conocer las características del agua utilizada en los tratamientos, primero se realizó un análisis del agua residual a ser usada para el riego de la alfalfa, determinando los valores de nitratos, nitritos, amonio, fósforo reactivo, sólidos, conductividad, $\mathrm{pH}$ y demanda química de oxigeno; como en Chamma (2002) (Tabla 1).

En segundo lugar, un análisis del agua obtenida por perforación (o de pozo) para el riego obteniendo valores de carbonatos, bicarbonatos, cloruros, sulfatos, calcio, magnesio, potasio, sodio, conductividad eléctrica, $\mathrm{pH}$ y relación de absorción de sodio (Tabla 2).

Para la determinación de la biomasa producida de alfalfa se realizaron muestreos al $10 \%$ de floración, obteniendo un total de 9 muestras por tratamiento de $0,35 \mathrm{~m}^{2}$ cada una. Se cortó con tijera al ras del suelo, separando cualquier tipo de material 
Tabla 1. Determinación analítica del agua residual urbana usada para el riego del cultivo de alfalfa

\begin{tabular}{lcc}
\hline \multicolumn{1}{c}{ Determinación } & Unidades & Valor \\
Nitratos $\left(\mathrm{NO}_{3}^{-}\right)$ & $\mathrm{mg} \mathrm{L}^{-1}$ & 18,30 \\
Nitritos $\left(\mathrm{NO}_{2}^{-}\right)$ & $\mathrm{mg} \mathrm{L}^{-1}$ & 0,05 \\
Amonio $\left(\mathrm{NH}_{4}^{+}\right)$ & $\mathrm{mg} \mathrm{L}^{-1}$ & 0,65 \\
Fósforo Reactivo $\left(\mathrm{PO}_{4}^{-3}\right)$ & $\mathrm{mg} \mathrm{L}^{-1}$ & 40 \\
Sólidos Totales Disueltos (STD) & $\mathrm{mg} \mathrm{L}^{-1}$ & 511 \\
Conductividad $18,4^{\circ} \mathrm{C}$ & $\mathrm{dS} \mathrm{m}^{-1}$ & 1046 \\
pH & & 8,01 \\
Demanda Química de Oxigeno (DQ0) & $\mathrm{mg} \mathrm{O}_{2} \mathrm{~L}^{-1}$ & 879 \\
\hline
\end{tabular}

Tabla 2. Determinación analítica del agua de perforación, usada para el riego del cultivo de alfalfa

\begin{tabular}{lcc}
\hline \multicolumn{1}{c}{ Determinación } & Unidades & Valor \\
Carbonatos & $\mathrm{mg} \mathrm{L}^{-1}$ & 0,0 \\
Bicarbonatos & $\mathrm{mg} \mathrm{L}^{-1}$ & 178,12 \\
Cloruros & $\mathrm{mg} \mathrm{L}^{-1}$ & 8,51 \\
Sulfatos & $\mathrm{mg} \mathrm{L}^{-1}$ & 16,57 \\
Calcio & $\mathrm{mg} \mathrm{L}^{-1}$ & 40,08 \\
Magnesio & $\mathrm{mg} \mathrm{L}^{-1}$ & 2,918 \\
Potasio & $\mathrm{mg} \mathrm{L}^{-1}$ & 19,55 \\
Sodio & $\mathrm{mg} \mathrm{L}^{-1}$ & 10,557 \\
Conductividad eléctrica (CE) & $\mathrm{dS} \mathrm{m}^{-1}$ & 0,45 \\
pH & & 7,4 \\
Relación de adsorción de sodio (RAS) & & 1,21 \\
\hline
\end{tabular}

extraño que no fuera propio del cultivo. Luego se procedió a la identificación de las muestras, que fueron secadas en estufa a una temperatura de $100{ }^{\circ} \mathrm{C}$ hasta peso constante. Las fechas en que se realizaron los diferentes cortes fueron: 19/12/07,31/ 01/08, 08/04/08 y 25/06/08. Hacía el final de la estación de crecimiento de la alfalfa (otoño) y cuando hubo disponibilidad del laboratorio para el análisis de las muestras (tercer corte) se extrajo material vegetal para hacer el análisis de calidad: energía metabólica, proteína bruta, fibra detergente neutra y fibra detergente acida. Este material se seco a $75^{\circ} \mathrm{C}$ en estufa y se entrego al laboratorio.

Un cuarto componente que se sumo al análisis fue tomar una muestra más de la parcela de alfalfa regada con agua residual urbana, y dejar que la deshidratación ocurriese de forma natural, esto con la intención de representar las condiciones de campo durante la confección del heno.

La comprensión de los procesos fisiológicos a campo, que determinan los flujos de agua en las plantas, coloca la eficiencia del uso de agua dentro de la administración adecuada de los recursos hídricos; y es por eso, que antes de hacer cualquier análisis sobre eficiencia, se hace necesario conocer el agua necesaria para el desarrollo del cultivo y su disponibilidad (riego, lluvia, nieve, corrientes subterráneas, entre otros), la capacidad de almacenamiento del agua en el suelo (textura, potencial hídrico y porosidad del suelo), la densidad y la profundidad del sistema radicular del cultivo; factor este, determinante en lo que respecta a la disponibilidad real de agua (Medrano et al., 2007, França et al., 2009).

La biomasa producida por unidad de agua consumida, expresa la eficiencia con la cual un cultivo fija carbono en relación al agua que pierde, y es a través de ella que medimos la eficiencia en el uso del agua (EUA). Para su determinación se utilizaron las siguientes ecuaciones (Martellotto et al., 2004):

$$
\mathrm{EUA}=\frac{\mathrm{B}_{\mathrm{p}}}{\mathrm{ET}_{\mathrm{r}}}
$$

$\mathrm{B}_{\mathrm{p}}$ - representa la biomasa aérea producida, kg MS Ha

$E T_{r}$ - la evapotranspiración real del cultivo, mm, calculada de manera técnica por

$$
\mathrm{ET}_{\mathrm{r}}=\mathrm{R}_{\mathrm{i}}+\mathrm{P}_{\mathrm{e}} \pm\left(\mathrm{L}_{\mathrm{f}}-\mathrm{L}_{\mathrm{i}}\right)
$$

$\mathrm{R}_{\mathrm{t}}$ - representa el riego estimado, $\mathrm{mm}$

$\mathrm{P}_{\mathrm{e}}$ - la precipitación efectiva, ocurridas y escorrentías en $\mathrm{mm}$

$\mathrm{L}_{\mathrm{f}}$ - la lámina final de agua, $\mathrm{mm}$

$\mathrm{L}_{1}$ - la lámina inicial de agua en el suelo, $\mathrm{mm}$

Una manera de cuantificar el potencial alimenticio de un forraje es a través del valor nutritivo relativo (VNR). En la actualidad, este índice es ampliamente usado para balancear raciones y fijar precios de comercialización del heno. Combinando las estimativas de los valores de digestibilidad de materia seca y consumo voluntario de materia seca (Goering $\&$ van Soest, 1970), es posible estimar de forma razonable el VNR, usando la fórmula:

$$
\begin{aligned}
\mathrm{VNR} & =[(88,9-(0,779 \times \mathrm{FDA} \%)) \times \\
& \times((120 \div \mathrm{FDN} \%) \div 1,29)]
\end{aligned}
$$

FDA - es la fibra detergente ácida

FDN - es la fibra detergente neutra

Undersander et al. (2006), proponen una categorización del heno de alfalfa en base a su valor nutritivo relativo: (excelente $>151$, primera $125-150$, segunda $103-124$, tercera $87-102$, cuarta $75-86$ y quinta $<75$ ).

En relación al análisis estadístico de los resultados obtenidos, se tomo la producción de biomasa aérea como la variable de mayor importancia, y se usó el programa estadístico Infostat para el análisis de varianza (ANAVA). En aquellos casos donde hubo diferencias de medias, se efectuó el método de comparaciones múltiples (DGC). El modelo estadístico de producción de biomasa aérea del cultivo de alfalfa usado en este ensayo, se encuadra en un diseño de bloques completos al azar con 9 repeticiones para cada uno de los tratamientos (Steel \& Torrie, 1997), regidos por la ecuación de producción:

$$
\mathrm{Y}_{\mathrm{ij}}=\mu+\alpha_{\mathrm{i}}+\mathrm{B}_{\mathrm{j}}+\varepsilon_{\mathrm{ij}}
$$

donde:

i - tratamientos - riego con agua de origen residual urbano, de perforación o de lluvias

$\mathrm{j}$ - bloques completos considerados

$\mathrm{y}_{\mathrm{ij}}$ - biomasa aérea del cultivo de alfalfa (Medicado sativa L.) obtenida en la unidad experimental que pertenece al bloque j con tratamiento i $\left(\mathrm{kg} \mathrm{MS} \mathrm{ha}^{-1}\right)$ 
$\mu \quad$ - media poblacional de la biomasa aérea $\left(\mathrm{kg} \mathrm{MS} \mathrm{ha}^{-1}\right)$

$\alpha_{i}$ - efecto debido al tratamiento i (riego)

$\mathrm{B}_{\mathrm{j}}$ - efecto debido al bloque $\mathrm{j}$

$\varepsilon_{\mathrm{ij}}$ - variable aleatoria debida al error en la unidad experimental que pertenece al bloque $\mathrm{j}$ con tratamiento i. Esta variable tiene $\sim \mathrm{N}\left(0 ; \sigma^{2}\right)$.

\section{RESULTADOS Y DISCUSIÓN}

Los resultados evidenciaron diferencias estadísticas entre los distintos tratamientos aplicados (riego con agua de origen residual urbano, de perforación o de lluvias) en cada una de las fechas de corte y con valores de probabilidad inferior o igual a 0,05 (Tabla 3).

Tabla 3. Producción de biomasa aérea de alfalfa, con distintos tratamientos de agua

\begin{tabular}{|c|c|c|c|c|}
\hline \multicolumn{5}{|c|}{ Producción de biomasa aérea por corte (kg MS ha $\left.{ }^{-1}\right)$} \\
\hline Tratamiento & $1^{\circ}$ Corte & $2^{\circ}$ Corte & $3^{\circ}$ Corte & $4^{\circ}$ Corte \\
\hline $\begin{array}{l}\text { Secano } \\
\quad \text { (precipitaciones) }\end{array}$ & $2899,56 b$ & $2820,09 \mathrm{~b}$ & $2366,54 \mathrm{c}$ & $2663,46 b$ \\
\hline $\begin{array}{l}\text { Riego con agua } \\
\text { de perforación }\end{array}$ & $3082,37 \mathrm{~b}$ & $3101,05 b$ & $2800,76 \mathrm{~b}$ & $2901,05 b$ \\
\hline $\begin{array}{l}\text { Riego con agua } \\
\text { residual urbana }\end{array}$ & 3303,65 a & 4100,22 a & $3479,45 a$ & $3295,49 a$ \\
\hline $\mathrm{R}^{2}$ & 0,32 & 0,41 & 0,69 & 0,61 \\
\hline C.V. & 9,94 & 22,18 & 12,00 & 8,16 \\
\hline Probabilidad & 0,0365 & 0,0037 & 0,0001 & 0,0001 \\
\hline
\end{tabular}

Letras distintas en la misma columna, indican diferencias significativas $(p \leq 0,05)$

En cada uno de los cortes, la mayor producción de alfalfa fue lograda por el tratamiento regado con agua residual urbana. No es posible inferir que hubo diferencias entre el tratamiento de riego con agua de perforación y el testigo, lo que si se observa es una tendencia a mayor rendimiento del primero, aunque estas diferencias no sean significativas. En los casos de primero, segundo y cuarto corte, las diferencias estadísticas entre regar y no regar con agua de perforación no son significativos; pero si lo son con respecto al riego con agua residual, donde tenemos la presencia de nutrientes. En el tercer corte, el análisis estadístico reconoce tres situaciones (señaladas con distintas letras), una de menor producción sin riego, separándola de las otras dos experiencias que recibieron riego y que estadísticamente fueron diferentes, probablemente por la presencia de nutrientes en el agua residual urbana.

Para conocer el efecto de los tratamientos sobre la producción total de biomasa aérea del cultivo de alfalfa (ciclo de producción), se sumaron las producciones de materia seca de los cuatro cortes (Tabla 4).

Los tratamientos de riego tuvieron un efecto positivo sobre la producción de biomasa aérea del cultivo en cuestión, encontrándose diferencias estadísticamente significativas. Por ejemplo, en las experiencias con riegos, se observo en promedio, un $24 \%$ más de producción de biomasa que en la situación de secano (precipitaciones). En cuanto a los tratamientos con riego, también se manifestaron diferencias estadísticamente significativas en la producción, un $19 \%$ superior a favor del riego con agua residual urbana.
Tabla 4. Producción total de biomasa aérea de alfalfa, con distintos tratamientos de agua. Materia seca acumulada

\begin{tabular}{lc}
\hline \multicolumn{1}{c}{ Tratamiento } & Medias $\left(\mathbf{k g ~ M S ~ h a} \mathbf{~}^{-1}\right)$ \\
Sin riego & $10749,65 \mathrm{C}$ \\
Riego con agua de perforación & $11885,22 \mathrm{~b}$ \\
Riego con agua residual urbana & $14178,81 \mathrm{a}$ \\
$\mathrm{R}^{2}$ & 0,77 \\
$\mathrm{C} . \mathrm{V}$. & 7,04 \\
Probabilidad & 0,0001 \\
\hline Letras distintas indican diferencias significativas $(\mathrm{p} \leq 0,05)$ &
\end{tabular}

En relación a los resultados obtenidos sobre la eficiencia en el uso del agua (EUA) (Tabla 5), los resultados indican que la EUA en secano fue mayor a la EUA con riego de perforación, y que hay poca diferencia con la EUA en riego con aguas residuales urbanas; indicando que el riego con aguas residuales es tan eficiente como el testigo o viceversa.

Tabla 5. Evapotranspiración real del cultivo (mm) y eficiencia en el uso de agua ( $\mathrm{kg} \mathrm{MS} \mathrm{ha}^{-1}$ ) usando agua residual urbana, de perforación y en secano. UNRC

\begin{tabular}{lcc}
\hline \multicolumn{1}{c}{ Tratamientos } & ETr $(\mathbf{m m})$ & EUA $\left(\mathbf{k g ~ M S ~} \mathbf{~ h a}^{-\mathbf{1}}\right.$ ) \\
Riego con agua residual urbana & 927,40 & 15,29 \\
Riego con agua de perforación & 887,90 & 13,38 \\
Secano & 672,40 & 15,98 \\
\hline
\end{tabular}

La baja eficiencia del uso del agua manifestada en los tratamientos de riego con respecto al testigo, puede deberse a la falta de agua de riego en el periodo estival. Sin embargo, la explicación es otra cuando se refiere solamente a los tratamientos con riego, donde las diferencias en EUA se deben a la calidad del agua de riego (Tabla 1), que tienen principalmente, los niveles de fosfatos $\left(\mathrm{PO}_{4}^{-3}\right)$ y nitratos $\left(\mathrm{NO}_{3}^{-}\right)$necesarios para el crecimiento de la alfalfa (Souza et al., 2010).

Muestras del material vegetal del tercer corte fueron llevadas al laboratorio, procurando comparar el valor nutritivo de cada condición de crecimiento (agua residual urbana, agua de perforación y sin riego). La Tabla 6 muestra los resultados obtenidos. Debemos recordar que los cultivos irrigados con efluentes de aguas residuales urbanas que contengan una carga alta de microorganismos, representan un riesgo para la salud pública, estos alimentos no deben ser consumidos crudos, deben ser deshidratados naturalmente antes de su consumo (Mara et al., 1990; Fasciolo et al., 2005).

Los resultados de la Tabla 6 muestran que la alfalfa regada con agua residual supera los valores nutritivos del cultivo que

Tabla 6. Análisis del valor nutritivo relativo de alfalfa en las condiciones de riego con agua residual, de perforación y sin riego

\begin{tabular}{lcccc}
\hline \multirow{2}{*}{$\begin{array}{c}\text { Fracciones } \\
\text { (en base seca) }\end{array}$} & \multicolumn{4}{c}{ Tratamientos } \\
\cline { 2 - 5 } & $\begin{array}{c}\text { Agua } \\
\text { Residual (AR) }\end{array}$ & $\begin{array}{c}\text { Agua de } \\
\text { perforación }\end{array}$ & Sin riego & $\begin{array}{c}\text { Deshidratada } \\
\text { naturalmente }\end{array}$ \\
PB (\%) & 28,51 & 20,01 & 20,48 & 19,39 \\
DQ (\%) & 60,65 & 57,97 & 53,31 & 56,65 \\
LDA (\%) & 5,74 & 7,07 & 8,47 & 7,11 \\
EM (Mcal kg-1 MS) & 2,19 & 2,09 & 1,92 & 2,04 \\
\hline PB - Proténa bruta; DQ - Digestibilidad química; LDA - Lignina detergente acida; EM - Energía
\end{tabular}
metabólica 
crece en condiciones normales de campo (sin riego); $39 \%$ más de proteínas (PB) y 14\% más de digestibilidad (DQ) y energía metabólica (EM).

En el caso de la muestra de alfalfa deshidratada, se observa que los valores de las cuatro fracciones son semejantes a los que tendría el forraje que crece en secano, e incluso mejora en digestibilidad. Este dato es importante al indicar que se puede obtener un forraje deshidratado para la confección de henos, con características semejantes a las de un forraje en pie.

Por último, utilizando la Ec. 3, se determinó el VNR para las cuatro muestras que se llevaron al laboratorio, y se clasificaron; en un forraje de primera para alfalfa regada con agua residual urbana $(\mathrm{VNR}=145,81)$ y de perforación $(\mathrm{VNR}=137,93)$ y un forraje de segunda para el caso de la alfalfa sin riego y muestra deshidratada (VNR $=122,7$ y VNR $=121,12$, respectivamente). Se observó que el proceso de deshidratación requerido para eliminar la presencia de microorganismos coliformes no disminuye la calidad del forraje.

\section{Conclusiones}

1. La mayor producción del cultivo de alfalfa (Medicago sativa L.) se obtuvo cuando se regó con agua residual urbana en relación al riego con agua de perforación y al cultivo en situación de secano. Esta producción se dio en cada uno de los cortes y en cada período de crecimiento del cultivo.

2 . El riego con aguas residuales de origen residencial produjo un incremento en la eficiencia del uso del agua por parte del cultivo.

3. El mayor valor del índice nutritivo relativo se obtuvo en el escenario del riego con aguas residuales urbanas. Destacándose a su vez, una buena calidad de forraje cuando este es deshidratado.

4. El buen uso del agua resultante del sistema integrado de tratamiento y uso de aguas residuales en la UNRC, contribuyo, significativamente con la producción y el valor nutritivo de la alfalfa.

\section{LITERATURA CITADA}

Álvarez, D. B.; Ramos, S. C.; Varaldo, H. P.; Sistemas de tratamiento de aguas residuales por aplicación al suelo. Avance y Perspectiva, v.21, p.333-340, 2002.

Basigalup, D. H. (ed.) El cultivo de la alfalfa en la Argentina. Buenos Aires: Ediciones INTA. 2007.476p.

Cabral, J. R.; Freitas P. S. L. de; Rezende R.; Muniz, A. S.; Bertonha, A. Impacto da água residuária de suinocultura no solo e na produção de capim elefante. Revista Brasileira de Engenharia Agrícola e Ambiental, v.15, p.823-831, 2011.

Chamma, M. D. L. Composição química de águas residuárias provenientes de efluentes urbanos destinadas à irrigação. Botucatu: FCA/UNESP, 2002. 84p. Dissertação Mestrado

Collino, D. J.; Dandanelli, J. L.; Luca, M. J. de; Racca, R. W. Temperature and water availability effects on radiation and water use efficiencies in alfalfa (Medicago Sativa L.) Australian Journal of Experimental Agriculture, v.45, p.383$390,2005$.
Fasciolo, G.; Calderón E.; Meca, M. I.; Contaminación microbiológica en ajos y suelos regados con efluentes domésticos tratados. Mendoza (Argentina). Revista FCA UN Cuyo. Tomo XXXVII.n.1, p.31-40. 2005.

França, J. X. de; Azevedo, C. A. V. de; Macêdo, N. E. B. de; Santos, A. R. de A.; Antunes, V. L. de L. Crescimento de mamoneira sob diferentes tipos de águas residuárias e níveis de água no solo. Revista Ambiente \& Água, v.4, p.196-211, 2009.

Goering, H. K.; Soest, P. J. van; Forage fiber analyses (apparatus, reagents, procedures and some applications). Washington: USDA, 1975. 20p. Agriculture Handbook, n.379

Leite D. V.; Athayde Júnior, G. B.;Sousa, J. T. de; Lopes W. S.; Prasad S.; Silva A. S.; Tratamento de águas residuárias em lagoas de estabilização para a aplicação na fertirrigação. Revista Brasileira de Engenharia Agrícola e Ambiental, v.9, p.71-75, 2005.

Maggi, C. F.; Freitas, P. S. L. de; Sampaio, S. C.; Dieter, J. Liviação de nutrientes em solo cultivado com aplicação de água residuária de suíno cultura. Revista Brasileira de Engenharia Agrícola e Ambiental, v.15, p.170-177, 2011.

Mara, D.; Cairncross, S.; Directrices para el uso sin riesgos de aguas residuales y excretes en agricultura y acuicultura. Ginebra: Publicadas por la Organización Mundial de la Salud en colaboración con el Programa de las Naciones Unidas para el Medio Ambiente, 1990. 210p.

Martellotto, E; Salas, P.; Lovera, E.; Salinas, A.; Giubergia, J. P.; Lingua, S.; Planilla de balance hídrico para riego. Proyecto Regionales: Agricultura sustentable, Gestión Agroambiental. Manfredi: INTA, EEA 2004. 9p.

Medrano, H; Bota, J.; Cifre, J.; Flexas, J.; Ribas Carból, M.; Gulías, J.; Eficiencia en el uso del agua por las plantas. Investigaciones Geográficas, v.43, p.63-84. 2007.

Pereira, M. M da S.; Sousa Tavares J. de S.; Ceballos, B. S. O.; Silva, B. F. da; Duarte, V. L. Tratamento aeróbico conjugado de lodos de tanques séticos e resíduos sólidos orgânicos domiciliares. Revista Ambiente \& Água, v.4, p.123-143, 2009.

Santos J. S. dos; Lima V., L. A. de; Borges Junior, J. C. F.; Silva L. V. B. D.; Azevedo, C. A. V. de. Mobilidade de solutos em colunas de solo com água residuária doméstica e de suinocultura. Revista Brasileira de Engenharia Agrícola e Ambiental, v.14, p.1226-1233, 2010.

Santos, V. B. dos; Matos A. T. de; Martinez M. A. ; Pereira M. P. F. Tratamentos de águas residuárias da suinocultura utilizando-se filtros orgânicos. Revista Brasileira de Engenharia Agrícola e Ambiental, v.4, p.327-333, 2000.

Schnoor, J.; Light, L.; Maccuttcheon, S.; Wolfe, N. L.; Carreira, L. Phytoremediation of organic and nutrient contaminants. Environmental Science and Technology, v.29, p.1-6. 1995.

Silva, M. B. R.; Fernandes, P. D.; Dantas Neto, J.; Nery A. R.; Rodrigues, L. N.; Viégas R. A. Crescimento e produção do pinhão-manso irrigado com água residuária sob condições de estresse hídrico. Revista Brasileira de Engenharia Agrícola e Ambiental, v.15, p.621-629, 2011. 
Souza, O. ; Federizzi, M.; Coelho, B.; Wagner, T. M.; Wisbeck, E. ; Biodegradação de resíduos lignocelulósicos generados na bananicultura e sua valorização para a produção de biogás; Revista Brasileira de Engenharia Agrícola e Ambiental, v.14, p. 438-443, 2010.
Steel, R.; Torrie, J. H.; Bioestadística: Principios y procedimientos. 2.ed. Tauscon: Mc Graw-Will. 1997. 622p. Undersander, D.; Jarvik, K; Anderson, T.; Milligan, L.; Schneider, N. Soybean silage. Wisconsin: Wisconsin Crop Manager, v.13, 2006. 235p. 